Pacific Journal of Mathematic 


\title{
DECOMPOSITION SPECTRA OF RINGS OF CONTINUOUS FUNCTIONS
}

\author{
Carl W. Kohls
}

\begin{abstract}
Let $S$ be a subset of a completely regular Hausdorff space $X$. Sufficient conditions on $S$ and $X$ are obtained for the ring of continuous real-valued functions on $S$ to be isomorphic to an inverse limit of quotient rings of the ring of continuous functions on $X$, or, alternatively, of the ring of bounded continuous functions on $X$. An application to the theory of rings of quotients of rings of continuous functions is given.
\end{abstract}

A decomposition spectrum of a set with some kind of structure is an inverse system of quotient structures of the same type. Decomposition spectra have been discussed recently by various authors: For topological spaces by Flachsmeyer [2], Pasynkov [4], and Vegrin [6]; and for ordered sets by Rinow [5]. Vegrin also considers briefly decomposition spectra of rings of continuous functions; however, the question he investigates is different from those considered here.

Definition. A decomposition spectrum of a ring $A$ is an inverse system of quotient rings of $A$.

The ring of all continuous real-valued functions on a completely regular Hausdorff space $X$ will be denoted by $C(X)$, and the subring of bounded functions by $C^{*}(X)$.

The inverse limit of a decomposition spectrum of a ring is, of course, a ring. In the papers on decomposition spectra mentioned above, it often turns out that the inverse limit is an extension of the original structure (topological space or ordered set). Now if $S$ is a subset of $X, C(S)$ is often an extension of $C(X)$; and $C(X)$ is always an extension of $C^{*}(X)$. This suggests the following questions: (1) For a subset $S$ of $X$, when is $C(S)$ isomorphic to the inverse limit of some decomposition spectrum of $C(X)$ ? (2) When is $C(X)$ isomorphic to the inverse limit of some decomposition spectrum of $C^{*}(X)$ ?

The first question has the trivial answer: When $S$ is $C$-embedded in $X$, that is when every function in $C(S)$ can be extended to a function in $C(X)$; for then, in fact, $C(S)$ is isomorphic to a quotient ring of $C(X)$, since the restriction mapping is a homomorphism onto $C(S)$. (This observation also leads naturally to the first question.) So some particular answers are: When $S$ is compact, or when $S$ is closed and $X$ is normal, or when $S$ is open-and-closed. The second question has the trivial answer: When $X$ is compact; for in that case, 
$C(X)=C^{*}(X)$. We show below that there are some nontrivial answers to both questions.

For any $f \in C(X)$ and any subset $B$ of $X$, the restriction of $f$ to $B$ will be designated by $f \mid B$, and the image of $B$ under $f$ will be written $f[B]$. The constant function in $C(X)$ whose value is $r$ will be denoted by $r$, and the greatest lower bound of $f$ and $g$ in the lattice $C(X)$ will be symbolized by $f \wedge g$. When we say that a collection $\left\{B_{r}\right\}$ of subsets of a space $Y$ containing a point $p$ determines the topology of $Y$ at $p$, we mean that if $f$ is a real-valued function on $Y$ and $f \mid B_{r}$ is continuous for all $B_{r}$, then $f$ is continuous at $p$.

The following lemma is used in obtaining all of our results on decomposition spectra. A parallel statement to the one given explicitly is indicated by the symbols in square brackets.

Lemma. Let $S$ be a subset of a completely regular Hausdorff space $X$. Suppose there exists a collection $\left\{T_{r}\right\}$ of subsets of $S$ with the following properties:

(1) $\left\{T_{r}\right\}$ is closed under finite unions;

(2) For each $p \in S$, the collection of all sets in $\left\{T_{r}\right\}$ containing $p$ determines the topology of $S$ at $p$;

(3) For each $f \in C(S)$ and each $T_{r}$, the function $f \mid T_{r}$ can be extended to a function in $C(X)\left[C^{*}(X)\right]$.

Then $C(S)$ is isomorphic to the inverse limit of a decomposition spectrum of $C(X)\left[C^{*}(X)\right]$.

Proof. One obtains the proof of the parallel statement by replacing " $C(X)^{\prime}$ " with " $C^{*}(X)^{\prime \prime}$ throughout the following proof.

From (1), $\left\{T_{\gamma}\right\}$ is directed by the relation $\supset$. For each $\gamma$, let $I_{\gamma}$ be the ideal $\left\{h \in C(X): h\left[T_{\gamma}\right]=\{0\}\right\}$. Now each $C(X) / I_{\gamma}$ is isomorphic to $\left\{g \mid T_{\gamma}: g \in C(X)\right\}$, so we shall view each element of $C(X) / I_{\gamma}$ as an element of $\left\{g \mid T_{r}: g \in C(X)\right\}$. Thus, if $T_{r} \supset T_{\delta}$, then the natural homomorphism defined by $g\left|T_{r} \rightarrow g\right| T_{\delta}$ for $g \in C(X)$ may be considered a homomorphism of $C(X) / I_{\gamma}$ onto $C(X) / I_{\delta}$. Also, the transitivity property is clearly satisfied by restriction mappings. Hence $\left\{C(X) / I_{r}\right\}$ and the natural homomorphisms comprise a decomposition spectrum of $C(X)$.

Now let $f \in C(S)$ be given. We define an element $\left(f_{\gamma}\right) \in \lim \left(C(X) / I_{\gamma}\right)$ as follows: For each $\gamma, f_{\gamma}$ is the image in $C(X) / I_{\gamma}$ of a $\overleftarrow{\text { function in }}$ $C(X)$ whose restriction to $T_{r}$ coincides with $f \mid T_{r}$; the existence of such a function is ensured by (3). Then $\left(f_{r}\right) \in \lim \left(C(X) / I_{r}\right)$, because $T_{r} \supset T_{\delta}$ implies $f_{r}\left|T_{\delta}=\left(f \mid T_{r}\right)\right| T_{\delta}=f \mid T_{\delta}=f_{\delta}$. The mapping $\sigma: f \rightarrow\left(f_{r}\right)$ embeds $C(S)$ in $\lim \left(C(X) / I_{\gamma}\right)$, since $f \neq g$ implies $f(p) \neq g(p)$ for some $p \in S$, whence $f_{r} \stackrel{\rightleftarrows}{\neq}$. for any $\gamma$ such that $p \in T_{\gamma}$. Furthermore, $\sigma$ is 
an isomorphism, because $(f+g)_{r}=f_{\gamma}+g_{\gamma}$ and $(f g)_{\gamma}=f_{\gamma} g_{\gamma}$ for each $\gamma$.

To prove that $\sigma$ is surjective, let $\left(b_{\gamma}\right) \in \lim \left(C(X) / I_{\gamma}\right)$ be given. Then $b_{\gamma}$ is the restriction of a function in $C(\overleftarrow{X})$ to $T_{\gamma}$, and, since $\gamma<\delta$ implies that $b_{\gamma}$ maps to $b_{\delta}$ under the natural homomorphism, $b_{\gamma}$ is an extension of $b_{\delta}$. By (2), $\left\{T_{\gamma}\right\}$ covers $S$, so $\left(b_{\gamma}\right)$ may be associated with a function $b$ on $S$. Since $b$ is continuous on each $T_{r}$, (2) implies that $b \in C(S)$; and $\sigma(b)=\left(b_{r}\right)$.

THEOREM 1. If $X$ is a first countable space and $S$ is any subset of $X$, then $C(S)$ is isomorphic to the inverse limit of a decomposition spectrum of $C(X)\left[C^{*}(X)\right]$.

Proof. Let $\left\{T_{\gamma}\right\}$ be the collection of all subsets of $S$ consisting of a finite number of points of $S$ together with sequences converging to those points. It is clear that (1) holds; (2) holds because $X$, and hence $S$, is first countable; and (3) holds because each $T_{r}$ is compact. Hence the Lemma is applicable.

CoRollary 1. If $X$ is a first countable space, then $C(X)$ is isomorphic to the inverse limit of a decomposition spectrum of $C^{*}(X)$.

THEOREM 2. If $X$ is a locally compact space, and $S$ is any open subset of $X$, then $C(S)$ is isomorphic to the inverse limit of a decomposition spectrum of $C^{*}(X)$.

Proof. Let $\left\{T_{\gamma}\right\}$ be the collection of finite unions of some family of compact neighborhoods of the points of $S$. It is evident that (1) and (2) hold; and (3) holds because each $T_{r}$ is compact. Hence the Lemma is applicable.

Corollary 2. If $X$ is a locally compact space, then $C(X)$ is isomorphic to the inverse limit of a decomposition spectrum of $C^{*}(X)$.

THEOREM 3. If $X$ is any completely regular Hausdorff space, and $S$ is any open subset of $X$, then $C(S)$ is isomorphic to the inverse limit of a decomposition spectrum of $C(X)$.

Proof. Let $p \in S$. By complete regularity, there exists an $h_{p} \in C(X)$ such that $h_{p}[X-S]=\{0\}, h_{p}(p)=2$, and $\mathbf{0} \leqq h_{p} \leqq 2$. Hence the nonnegative function $g_{p}=h_{p} \wedge 1$ has the properties $g_{p}[X-S]=$ $\{0\}$ and $g_{p}\left[U_{p}\right]=\{1\}$, where $U_{p}$ is a neighborhood of $p$. Choose one such neighborhood for each point of $S$, and let $\left\{T_{r}\right\}$ be the collection 
of finite unions of these neighborhoods. Again, (1) and (2) are clear. To see that (3) holds, consider a particular $T_{r}=U_{p_{1}} \mathrm{U} \cdots \cup U_{p_{n}}$. The nonnegative function $g_{p_{1}}+\cdots+g_{p_{n}}$ is zero on $X-S$ and at least one on $T_{r}$, whence $g_{r}=\left(g_{p_{1}}+\cdots+g_{p_{n}}\right) \wedge 1$ has the properties $g_{r}[X-S]=\{0\}, g_{r}\left[T_{r}\right]=\{1\}$, and $0 \leqq g_{r} \leqq 1$. If $f \in C(S)$, we define $f_{\gamma} \in C(X)$ by $f_{\gamma}[X-S]=\{0\}$ and $f_{\gamma}(x)=\tan \left(\left(g_{\gamma}(x)\right)(\arctan (f(x)))\right)$ for $x \in S$. Then $f_{r}\left|T_{r}=(\tan \circ \arctan \circ f)\right| T_{r}=f \mid T_{r}$, as required. Thus (3) holds, and again the Lemma is applicable.

REMARK. If $S$ is a cozero-set in $X$, say $S=\{x \in X: h(x) \neq 0\}$, where $h \in C(X)$, then the decomposition spectrum can be formed from an $\omega^{*}$-sequence of quotient rings of $C(X)$. For each positive integer $n$, we set $T_{n}=\{x \in X:|h(x)| \geqq 1 / n\}$. There exists a function $g_{n} \in C(X)$ such that $g_{n}[X-S]=\{0\}, g_{n}\left[T_{n}\right]=\{1\}$, and $0 \leqq g_{n} \leqq 1$, since $T_{n}$ is completely separated from $X-S[3 ; 1.15]$. The proof that the collection $\left\{T_{n}\right\}$ satisfies (3) then concludes as in the proof of Theorem 3. Now (1) is evident, and (2) holds because each $p \in S$ is in the interior of some $T_{n}$; so the Lemma is applicable to this situation too.

We now give an application of Theorem 3. First recall that the maximal ring of quotients of a commutative ring $A$ with identity may be obtained as the direct limit of the $A$-homomorphisms of dense ideals of $A$ into $A[1 ; 1.9]$, and that the classical ring of quotients may be obtained similarly from the $A$-homomorphisms of dense principal ideals $[1 ; 1.10]$. Fine, Gillman, and Lambek have shown that (1) the maximal ring of quotents of $C(X)$ has a representation as the direct limit of rings $C(U), U$ ranging over the dense open sets in $X$, and that (2) the classical ring of quotients of $C(X)$ has a representation as the direct limit of rings $C(U), U$ ranging over the dense cozero-sets in $X[1 ; 2.6]$. Combining Theorem 3 with these facts yields the following result.

CoRollaRY 3. If $X$ is any completely regular Hausdorff space, theu both the maximal and classical rings of quotients of $C(X)$ have representations as a direct limit of inverse limits of quotient rings of $C(X)$.

\section{REFERENCES}

1. N. J. Fine, L. Gillman, and J. Lambek, Rings of quotients of rings of functions, Monograph form, McGill University Press, Montreal.

2. J. Flachsmeyer, Zur Spektralentwicklung topologischer Räume, Math. Ann. 144 (1961), 253-274.

3. L. Gillman and M. Jerison, Rings of Continuous Functions, van Nostrand, Princeton, 1960. 
4. B. A. Pasynkov, On spectral decomposition of topological spaces, Matemat. Sbornik 66 (1965), 35-79 (Russian).

5. W. Rinow, Zerlegungsspektren geordneter Mengen, Z. Math. Logik Grundlagen Math. 10 (1964), 331-360.

6. L. D. Vegrin, Direct spectra of topological spaces, Vestnik Moskov. Univ. Ser. I Mat. Meh. 1961, 20-24 (Russian).

Received January 3, 1966.

SyraCUSE UNIVERSITY, Syracuse, N. Y. 



\section{PACIFIC JOURNAL OF MATHEMATICS}

\section{EDITORS}

\section{H. ROYDEN}

Stanford University

Stanford, California

J. P. JANS

University of Washington

Seattle, Washington 98105

\section{J. DUGUNDJI}

Department of Mathematics

Rice University

Houston, Texas 77001

RICHARD ARENS

University of California

Los Angeles, California 90024

ASSOCIATE EDITORS

E. F. BECKENBACH

B. H. NeumanN

F. WoLF

K. YosidA

\section{SUPPORTING INSTITUTIONS}

\author{
UNIVERSITY OF BRITISH COLUMBIA \\ CALIFORNIA INSTITUTE OF TECHNOLOGY \\ UNIVERSITY OF CALIFORNIA \\ MONTANA STATE UNIVERSITY \\ UNIVERSITY OF NEVADA \\ NEW MEXICO STATE UNIVERSITY \\ OREGON STATE UNIVERSITY \\ UNIVERSITY OF OREGON \\ OSAKA UNIVERSITY \\ UNIVERSITY OF SOUTHERN CALIFORNIA
}

\author{
STANFORD UNIVERSITY \\ UNIVERSITY OF TOKYO \\ UNIVERSITY OF UTAH \\ WASHINGTON STATE UNIVERSITY \\ UNIVERSITY OF WASHINGTON \\ AMERICAN MATHEMATICAL SOCIETY \\ CHEVRON RESEARCH CORPORATION \\ TRW SYSTEMS \\ NAVAL ORDNANCE TEST STATION
}




\section{Pacific Journal of Mathematics}

\section{Vol. 23, No. 1 \\ March, 1967}

M. J. C. Baker, A spherical Helly-type theorem ................... 1

Robert Morgan Brooks, On locally m-convex*-algebras.............. 5

Lindsay Nathan Childs and Frank Rimi DeMeyer, On automorphisms of separable algebras ...................................

Charles L. Fefferman, A Radon-Nikodym theorem for finitely additive set

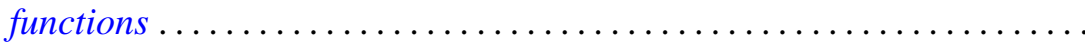

Magnus Giertz, On generalized elements with respect to linear

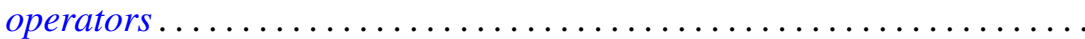

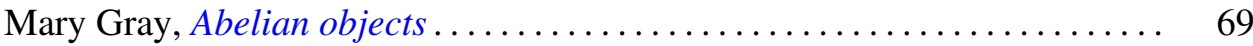

Mary Gray, Radical subcategories.............................. 79

John A. Hildebrant, On uniquely divisible semigroups on the two-cell . . . . . 91

Barry E. Johnson, AW*-algebras are $\mathrm{QW}^{*}$-algebras ............... 97

Carl W. Kohls, Decomposition spectra of rings of continuous functions . . . . 101

Calvin T. Long, Addition theorems for sets of integers .............. 107

Ralph David McWilliams, On $w^{*}$-sequential convergence and quasi-reflexivity ................................... 113

Alfred Richard Mitchell and Roger W. Mitchell, Disjoint basic

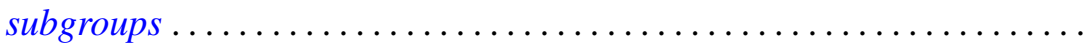

John Emanuel de Pillis, Linear transformations which preserve hermitian and positive semidefinite operators .

Qazi Ibadur Rahman and Q. G. Mohammad, Remarks on Schwarz's lemma

Neal Jules Rothman, An $L^{1}$ algebra for certain locally compact topological semigroups ...

F. Dennis Sentilles, Kernel representations of operators and their adjoints ...

D. R. Smart, Fixed points in a class of sets

K. Srinivasacharyulu, Topology of some Kähler manifolds

Francis C.Y. Tang, On uniqueness of generalized direct decompositions .

171 Albert Chapman Vosburg, On the relationship between Hausdorff dimension and metric dimension . 